\title{
Good blogosphere practices
}

\author{
A recent brouhaha over corporate-sponsored scientific blogs sharing the same platform as those that are editorially \\ independent highlights the need for clearly disclosing any potential conflicts of interest in the blogosphere.
}

T his July, a PepsiCo-sponsored blog at the popular science website Scienceblogs.com was canceled two days after its launch because of mounting discontent from other independent scientific bloggers. The blog, Food Frontiers, was to be written by food scientists employed by PepsiCo, highlighting the company's research. However, many bloggers at Scienceblogs.com, some of whom are practicing scientists and science journalists, felt that the scientific independence, integrity and reputation that they had built at Scienceblogs.com was being misused by Seed Media Group, the company that hosts the blog platform. Several bloggers left the site in protest and this incident led to heated discussions about practices of scientific blogging and whether commercial blogs ought to be allowed to share a blogging platform with blogs that attract science journalists and that are considered by some as an alternative news source. The Food Frontiers incident highlights the need for blogs that have evolved as news sources to denote their purposes and intentions clearly to their readers and for these alternate media outlets to clearly segregate independent scientific blogs from those that have a commercial agenda.

As with all blogs, science blogging started as a grass-roots movement to freely share opinions on scientific findings and rapidly exchange, publicize and discuss scientific matters. As the popularity of blogs has grown, however, some blog conglomerates, such as Scienceblogs.com or Discovery News (http://news.discovery.com), that invite a selected group of science bloggers to use their platform are being viewed as a 'news' source on the web; their content is indexed in the news aggregation web tool Google News. The decline of scientific journalism in the traditional mass media (both print and broadcast) has also fueled the importance of independent scientific blogs as disseminators of scientific information and catalysts for public scientific discussions; in $2009,63 \%$ of science journalists employed by mainstream media outlets reported to have found 'stories' on a scientist's blog, compared with $15 \%$ five years earlier ${ }^{1}$.

This evolution of the blogosphere, and especially certain large blog platforms, from servicing niche interests to now being seen (intentionally or unintentionally) as alternate news sources places an onus on these platforms to act with the same code of ethics as we would expect from respectable mainstream media outlets. The nature of blogging makes it especially easy to hide any secondary motivations that may lurk behind blog posts. Blogs can be anonymous and there is no explicit requirement on most sites that bloggers or commentators disclose their background or conflicts of interests before posting a story or comments. A casual reader surfing blogs for information can easily be confused and overwhelmed by the sheer amount of blogs, information and opinions and it can be very difficult for the average reader to fact-check the accuracy of a blog entry or divine the underlying motivation of the blogger. For this reason, many science-blog readers turn to managed blog networks such as Scienceblogs.com, where they expect a certain amount of credibility and integrity from expert bloggers that were specifically invited to contribute.

It is because of this expectation that blogs that have taken on the mantle of science journalists must be especially careful to not blur the lines between editorially independent content and advertisements or externally generated content. One problem with Food Frontiers was that Seed Media failed to make this demarcation especially clear because it hosted the corporate-sponsored blog, even with the advertorial disclaimer, on the same platform as the rest of the editorially independent blogs. The main issue with 'blogotisements' (advertisements formatted to look like a regular blog entries) is one of intention versus perception. In principle, using a blog to disseminate company-related information is perfectly fine. At the same time, the intent must be crystal clear (for example, by containing a conflict of interest statement and/or background information on who the bloggers are) so that readers can judge for themselves who is providing the information. Even with clear disclaimers, casual readers may incorrectly perceive the informational content as editorially independent because of the branding and reputation associated with the particular blog/publication platform. In most cases, reputable platforms such as ScienceBlogs.com and Nature Publishing Group's own Nature Network have no editorial input into the contents of the blog entries. Quality control relies on bloggers and readers self-policing the blog entries through their commenting. The initial invitation to host a scientific blog on a reputable platform bestows implicit credence to the bloggers and the editors and publishers of such platforms need to be especially careful to maintain this credence by ensuring that their motives and their criteria for inclusion are clearly indicated. Bloggers who are invited to join such platforms should be expected to adhere to the same ethical standards that we would expect from good journalists and blogotisements or commercial content should be strictly segregated from editorial posts.

We recognize that scientific blogs are a relatively new medium for the exchange of ideas that will be increasingly important in the way science is communicated among scientists and to the public and we acknowledge that some blogging platforms will be seen as a bona-fide source of science news. Particularly for these platforms, where the content is typically still not vetted by independent experts before being broadcast, it is important to ensure that good journalistic blogosphere practices are followed, that blogotisements are clearly labeled and segregated from regular blog posts and that conflicts of interest are clearly stated. Not doing so risks promoting the further erosion of public trust in science.

1. Brumfiel, G. Science journalism: supplanting the old media? Nature 458, 274-277 (2009). 